\title{
PERCEPÇÕES E EXPERIÊNCIAS DE TRABALHADORES DE ENFERMAGEM SOBRE O CUIDADO AO PACIENTE EM MORTE ENCEFÁLICA
}

\author{
PERCEPTIONS AND EXPERIENCES OF NURSING \\ WORKERS ABOUT PATIENT CARE IN BRAIN DEATH
}

\section{LAS PERCEPCIONES Y EXPERIENCIAS DE LOS TRABAJADORES DE ENFERMERÍA SOBRE EL CUIDADO DEL PACIENTE EN MUERTE ENCEFÁLICA}

\author{
Mariana Pellegrini Cesar ${ }^{1}$ \\ Silviamar Camponogara ${ }^{2}$ \\ Quezia Boeira da Cunha \\ Camila Pinno $^{4}$ \\ Nara Marilene Oliveira Girardon-Perlini ${ }^{2}$ \\ Cintia Lovato Flores ${ }^{5}$
}

\begin{abstract}
Como citar este artigo: Cesar MP, Camponogara S, Cunha QB, Pinno C, Girardon-Perlini NMO, Flores CL. Percepções e experiências de trabalhadores de enfermagem sobre o cuidado ao paciente em morte encefálica. Rev baiana enferm. 2019;33:e33359.

Objetivo: conhecer as percepções e experiências dos trabalhadores de enfermagem atuantes em terapia intensiva acerca do cuidado de pacientes com suspeita ou diagnóstico de morte encefálica. Método: pesquisa de abordagem qualitativa, de caráter exploratório-descritivo, realizada com 19 profissionais de enfermagem de um Hospital Universitário no interior do estado do Rio Grande do Sul, Brasil, por meio de entrevista semiestruturada e questionário sociodemográfico. Resultados: o cuidado com paciente em morte encefálica está permeado por problemáticas relativas ao desgaste emocional e por complexidade. Além disso, destacam-se as dificuldades na abordagem da família do potencial doador, bem como a falta de qualificação e preparo requeridos para o cuidado. Conclusão: o cuidado de enfermagem para com o paciente em morte encefálica está permeado por inúmeras dificuldades e enfrentamentos, resultando na necessidade de qualificação profissional e apoio psicológico para os trabalhadores.
\end{abstract}

Descritores: Profissionais de Enfermagem. Morte Encefálica. Obtenção de Tecidos e Órgãos. Cuidados de Enfermagem. Unidades de Terapia Intensiva.

Objective: to know the perceptions and experiences of nurse practitioners working in intensive care about the care of patients with known or suspected diagnosis of brain death. Method: qualitative, exploratory-descriptive study, performed with 19 nursing professionals from a University Hospital in the countryside of the state of Rio Grande do Sul, Brazil, by means of interviews and sociodemographic questionnaire. Results: the care with the patient in brain

\footnotetext{
Enfermeira. Especialista em Urgência e Trauma. Universidade Federal de Santa Maria. Santa Maria, Rio Grande do Sul, Brasil. marianapcesar@hotmail.com. https:// orcid.org/0000-0002-7938-56/4

Enfermeira. Doutora em Enfermagem. Professora da Universidade Federal de Santa Maria. Santa Maria, Rio Grande do Sul, Brasil. https://orcid.org/0000-000 I -93423683; https://orcid.org/0000-0002-3604-2507

3 Enfermeira. Mestre em Enfermagem. Universidade Federal de Santa Maria. Enfermeira Assistencial no Hospital Universitário de Santa Maria. Santa Maria, Rio Grande do Sul, Brasil. https://orcid.org/0000-000 I-7014-9343

4 Enfermeira. Mestre em Enfermagem. Universidade Federal de Santa Maria. Santa Maria, Rio Grande do Sul, Brasil. http://orcid.org/0000-0002-9|45-1987

5 Enfermeira. Enfermeira Assistencial no Hospital Universitário de Santa Maria. Santa Maria, Rio Grande do Sul, Brasil. https://orcid.org/0000-000 I-6459-6454
} 
death is permeated by problems relating to the emotional distress and complexity. Furthermore, the difficulties to approach the potential donor's family stand out, as well as the lack of qualification and training required for the care. Conclusion: the nursing care with the patient in brain death is permeated by numerous difficulties and coping, resulting in the need for professional qualification and psychological support for workers.

Descriptors: Nurse Practitioners. Brain Death. Tissues and Organ Procurement. Nursing Care. Intensive Care Units.

Objetivo: conocer las percepciones y experiencias de los trabajadores de enfermería en cuidados intensivos sobre el cuidado de los pacientes con diagnóstico conocido o sospechoso de la muerte encefálica. Método: estudio cualitativo, exploratorio-descriptivo, realizado con 19 profesionales de enfermería de un Hospital Universitario en el interior del estado de Rio Grande do Sul, Brasil, por medio de entrevistas y cuestionario sociodemográfico. Resultados: el cuidado con el paciente en muerte encefálica está permeado por problemas relacionados con el estrés emocional y la complejidad. Además, se destacan las dificultades para abordar la familia del posible donante, así como la falta de calificación y preparación necesarias para el cuidado. Conclusión: la atención de enfermería en el paciente en muerte encefálica está permeada por numerosas dificultades y enfrentamiento, resultando en la necesidad de cualificación profesional y de apoyo psicológico para los trabajadores.

Descriptores: Enfermeras Practicantes. Muerte Encefálica. Obtención de Tejidos y Órganos. Atención de Enfermería. Unidades de Cuidados Intensivos.

\section{Introdução}

O conceito de morte evoluiu ao longo do tempo. Inicialmente, era definida como cessação dos batimentos cardíacos, porém, com o avanço tecnológico, a definição sobre a morte mudou, verificando-se que o indivíduo, mesmo morto, poderia manter alguns sinais vitais, quando ligado a aparelhos e privado da função cerebral. Assim, esses avanços permitiram a redefinição de um novo conceito: o de morte encefálica ${ }^{(1-2)}$.

A evolução do conceito de morte encefálica foi fundamental para que pudesse ocorrer a doação de órgãos de um paciente falecido, pois ela permite a manutenção das funções vitais até que os órgãos sejam retirados do doador. Para isso, a morte encefálica precisa ser diagnosticada por meio de critérios legais e pré-definidos $^{(1,3)}$. Quando diagnosticada em tempo hábil, ela possibilita a captação de órgãos, favorecendo o transplante, que se configura em uma única alternativa terapêutica frente às diversas doenças que causam insuficiência ou falência de órgãos ou tecidos ${ }^{(2)}$.

No Brasil, a morte encefálica é regulamentada pela Resolução n. 2.173/17, do Conselho Federal de Medicina (CFM) ${ }^{(4)}$, devendo ser diagnosticada por meio da realização de exames clínicos e complementares em intervalos de tempo variáveis. A morte encefálica poderá ser definida por apneia, ausência dos reflexos do tronco encefálico (mesencéfalo, ponte e bulbo), coma aperceptivo e perda irreversível das funções do encéfalo (cérebro e troco encefálico) ${ }^{(2-4)}$. Para isso, são necessárias a avaliação e a identificação correta e rigorosa do diagnóstico. Todos os profissionais de saúde têm papel fundamental nesse processo que, se bem desempenhado, pode aumentar substancialmente as doações de órgãos e tecidos para transplante ${ }^{(5)}$.

É no contexto da Unidade de Terapia Intensiva (UTI) que é mais presente a atuação da equipe de enfermagem junto a pacientes com morte encefálica. Normalmente, é nesse ambiente que o paciente tem confirmação de diagnóstico e passa por avaliação rigorosa de critérios clínicos, tornando-se um potencial doador de órgãos ${ }^{(5-6)}$.

Nesse setor, destaca-se a importância do papel da equipe de enfermagem, principalmente na manutenção rigorosa das funções vitais do potencial doador. Para tanto, é necessário que essa equipe tenha conhecimento técnico e científico qualificado acerca de todo o processo de cuidado ao paciente em morte encefálica, pois a conservação adequada influencia diretamente na viabilidade dos órgãos e tecidos a serem doados $^{(1,6-8)}$. A complexidade do cuidado a esse 
paciente, no entanto, vai além da necessidade de monitorização rigorosa dos aspectos biológicos, envolvendo também a família do potencial doador e a possível doação ${ }^{(8)}$.

No ano de 2018, a taxa de doadores efetivos cresceu apenas 2,4\% em relação ao ano anterior, ficando 5,5\% abaixo da taxa prevista. Ademais, o crescimento da taxa de transplante de órgãos com doador falecido foi somente $0,7 \%$, o que revela aumento na taxa de não utilização dos órgãos dos doadores falecidos. Em relação à taxa de não doação por doadores falecidos, destaca-se que, em 25\% dos casos, ocorre por recusa familiar $^{(9)}$. Frente a isso, destaca-se a necessidade de aprimoramento profissional acerca do tema, a fim de melhorar o panorama nacional de transplantes de órgãos, o reconhecimento precoce e a manutenção adequada dos potenciais doadores de órgãos.

Ressalta-se que os trabalhadores de enfermagem que atuam em terapia intensiva necessitam conhecer o processo de suspeita e diagnóstico de morte encefálica e o seu impacto para futuras doações. Entretanto, os estudos sobre morte encefálica são mais voltados para o processo da morte encefálica em si, não abordando especificamente os aspectos relacionados à atuação do profissional de enfermagem que está continuamente realizando o cuidado ${ }^{(6)}$. Por ser um tema recente e ainda pouco explorado, inclusive na formação acadêmica ${ }^{(10)}$, muitos trabalhadores podem ter dificuldades na condução do processo assistencial, especialmente por se tratar de um tema que envolve questões emocionais.

Pesquisas ${ }^{(6,11)}$ apontam que as diferentes percepções dos enfermeiros a respeito do cuidado com o paciente em suspeita ou diagnóstico de morte encefálica podem acabar afetando o processo de cuidado e interferir nas possibilidades para que aquele paciente torne-se um doador efetivo. O objetivo deste estudo foi conhecer as percepções e experiências dos trabalhadores de enfermagem atuantes em terapia intensiva acerca do cuidado de pacientes com suspeita ou diagnóstico de morte encefálica.

\section{Método}

Trata-se de uma pesquisa de abordagem qualitativa, de caráter exploratório-descritivo. O estudo foi desenvolvido em uma UTI adulto de um Hospital Universitário (HU) da Região Sul do Brasil, que dispõe de 10 leitos e atende pacientes clínicos e cirúrgicos, com diferentes condições patológicas e idade superior a 18 anos.

Os participantes do estudo foram trabalhadores de enfermagem (enfermeiros e técnicos de enfermagem). Foi estabelecido como critério de inclusão ter, no mínimo, um ano de atuação na unidade. Como critério de exclusão adotou-se estar em período de férias ou qualquer tipo de licença no período da produção de dados. Os participantes foram selecionados por meio de sorteio com base em uma lista disponibilizada pela instituição, visando evitar viés na pesquisa e para garantir a aleatoriedade dos profissionais selecionados. A proporcionalidade entre as diferentes categorias profissionais (enfermeiro e técnicos de enfermagem) e os turnos de trabalho foi considerada. A pesquisadora possuía um vínculo prévio com a equipe, tendo em vista a realização de seu estágio da graduação na unidade.

A produção de dados deu-se por meio de entrevista semiestruturada, com base em roteiro previamente construído, contendo questões atinentes ao objeto de estudo e com base na literatura. Foram elaboradas 10 questões abertas, a fim de possibilitar que o profissional articulasse suas percepções e experiências no cuidado dirigido a pacientes com suspeita ou diagnóstico de morte encefálica, como, por exemplo: Você já cuidou de pacientes com suspeita ou diagnóstico de morte encefálica? O que significou isso para você? Como você se sente cuidando de um paciente com suspeita ou diagnóstico de morte encefálica? Como você percebe o cuidado ao paciente com suspeita ou diagnóstico de morte encefálica na UTI?

Foi utilizado também um formulário para coleta de dados sociodemográficos e profissionais. Antes do período da coleta foram realizadas 3 entrevistas testes para avaliação do instrumento de 
coleta de dados e também para familiarização da entrevistadora, uma graduanda de enfermagem previamente capacitada. Foram entrevistados 19 trabalhadores de enfermagem, nos meses de julho e agosto de 2016. Não houve recusa de participação no estudo. O número final de participantes foi determinado pela necessidade de informação e qualidade dos dados produzidos, quando se alcançou a reincidência e a complementariedade das informações acerca do objeto de estudo ${ }^{(12)}$.

Os participantes foram abordados face a face, após sorteio, sendo informalmente convidados a participar do estudo. As entrevistas foram realizadas após o aceite do sorteado, durante o turno de trabalho, em uma sala de reuniões do setor, mediante leitura e assinatura do Termo de Consentimento Livre e Esclarecido (TCLE). A fim de preservar a privacidade dos participantes, permaneceram na sala apenas o pesquisador e o entrevistado. O tempo médio de duração das entrevistas foi de 20 minutos.

As entrevistas foram gravadas com aparelho digital (MP4 player) e posteriormente transcritas pela pesquisadora e identificadas por números cardinais sequenciais, conforme a ordem de realização e a categoria profissional de cada participante (E1, E2, E3, TE1, TE2, TE3...). Os dados não retornaram para os entrevistados antes da realização da análise.

A análise dos resultados seguiu o processo e os passos da análise de conteúdo proposta por Laurence Bardin ${ }^{(13)}$. Esse tipo de análise é composto por três fases: a pré-análise, momento em que se efetua a leitura flutuante do material, tomando-se contato de sua estrutura, seguida da constituição do Corpus, considerando a exaustividade, representatividade, homogeneidade e pertinência; na segunda fase é realizada a leitura em profundidade do material analisado, com vistas ao encontro de categorias para posterior classificação e agregação dos dados; na terceira fase, realiza-se o tratamento dos resultados e a interpretação dos dados ${ }^{(13)}$.

A presente pesquisa cumpriu as determinações da Resolução n. 466, de 12 de dezembro de 2012, do Conselho Nacional de Saúde ${ }^{(14)}$.
Foi submetida à apreciação do Comitê de Ética em Pesquisa da Universidade Federal de Santa Maria, obtendo aprovação sob n. 1.670.591. Seguiram-se as Diretrizes de Critérios Consolidados para Relatos de Pesquisa Qualitativa (COREQ) ${ }^{(15)}$.

\section{Resultados}

Foram entrevistados 19 trabalhadores de enfermagem, dentre eles, 6 enfermeiros $(31,6 \%)$ e 13 técnicos de enfermagem (68,4\%). Do total, $14(73,7 \%)$ participantes eram do sexo feminino e $5(26,3 \%)$ do sexo masculino. As idades variaram ente 25 e 56 anos. O tempo de atuação na instituição variou de 1 a 22 anos, e o tempo de atuação na unidade variou de 1 a 21 anos.

Após análise dos resultados, emergiu a categoria: "O cuidado ao paciente com suspeita ou diagnóstico de morte encefálica: implicações para os profissionais de enfermagem", a qual se subdivide em três subcategorias, descritas a seguir.

\section{Dificuldades no cuidado ao paciente com suspeita ou diagnóstico de morte encefálica}

Ao falar sobre suas experiências no trabalho com pacientes com diagnóstico ou suspeita de morte encefálica, os participantes relataram as dificuldades que enfrentavam diante da morte. Em suas falas surgiram expressões como "inutilidade" e "impotência" para descrever o cuidado ao paciente em morte encefálica, já que esse cuidado é prestado para um paciente sem possibilidade de vida.

\footnotetext{
Não me sinto confortável porque lidar com a doença, lidar com a morte é difícil para a gente [...] Na enfermagem a gente é formado para cuidar das pessoas a saírem com vida, não saírem com morte. (E6)

Inutilidade, sei lá, mas é mesmo estranbo tu estar cuidando de um paciente ali que pode acordar a qualquer momento, sair, mas esse daí não. (E7).

[...] é que é complicado, porque tu sabes que não tem o que fazer ali. Só tem que prestar os cuidados básicos, mas que ele não vai sair dali, não vai evoluir o quadro dele. Então, é uma situação de impotência mesmo. (TE7).

Os trabalhadores descreveram que os pacientes em morte encefálica eram, em sua
} 
maioria, jovens, o que, em muitos casos, gerava uma comoção entre os profissionais. Por outro lado, alguns participantes afirmaram buscar um distanciamento emocional, para conseguir lidar com os aspectos emocionais que envolviam esse cuidado, após a constatação de morte encefálica, como pode ser observado nos seguintes depoimentos:

Eu penso mais na situação da pessoa ali, ainda mais quando é jovem, que geralmente é traumatismo, alguma coisa assim. Eu pessoalmente fico mais emotiva, fico me questionando da vida, daquilo tudo sabe? [...] fica pensando que poderia ser tu ali. (TE8).

A gente tem que usar bastante a razão nesses casos, não se deixar levar pelo emocional. (TE12).

Para mim é normal, porque a gente vai adquirindo essa barreira de cuidar um pouco os sentimentos, não se apegar muito. Não que tu seja insensivel, mas tu aprende a criar essa barreira, e tu vai aprendendo a trabalbar este lado, que é uma pessoa sim, que tu vai tratar com todos os cuidados normais, só que tu sabe que ali não existe mais vida. (TE6)

Alguns depoimentos demonstraram que os trabalhadores ainda apresentavam dificuldades de compreender e/ou aceitar o diagnóstico de morte encefálica. Essa dúvida acabava mobilizando sentimentos, diante da contradição de haver uma condição definitiva de morte frente a um corpo que apresentava sinais de vida: movimentos respiratórios (mesmo que artificialmente), pressão arterial, pulso, calor, entre outros. Em relação a esse aspecto, surgiram as seguintes afirmações:

Aí, quando eu falo em morte encefálica, eu penso assim: "Meu Deus, será que é todo o cérebro mesmo? Será que ele está morto mesmo?" Porque a gente sabe que a morte é do encéfalo, daí a gente tem a ventilação mecânica que está correndo, o coração está batendo, está tudo viável [...] Mas a gente tem a medicina para ver que não tem mais movimento, de sinapses que não tem nada. É um cérebro morto, que não, que não vai falar mais, que não tem, que só está aguardando para morrer. Eu acho triste, eu acho tudo muito triste. (E6).

No momento, é estranbo tu estar cuidando de um corpo que tu sabes que está morto, mas, ao mesmo tempo, tu vês a pessoa ali, parecia dormindo. (TE13).

Os depoimentos dos participantes indicavam que, muitas vezes, existia uma grande preocupação com os cuidados de pacientes em morte encefálica devido à possibilidade da doação de órgãos. Isso pode ser percebido no relato a seguir, em que o foco do profissional muda daquele paciente que está na sua frente, com o possível diagnóstico de morte, para aquele outro paciente que está na fila de transplante, para preservar os órgãos, no caso de confirmação da doação. Além disso, a possibilidade de proporcionar vida normal àqueles que estão vulneráveis, aguardando na fila de transplantes, pode ser identificada como um fator de motivação para os profissionais que percebem a importância da doação de órgãos no cuidado prestado a esse paciente.

Eu sou mais melindrosa, mais cuidadosa, mais presente ainda, em função da preservação, se ele for um doador. Se ele não for um doador, ele já está morto. (TE12).

Aquele desejo que doe os órgãos [...] então, a gente cuida com todo cuidado, respeito que outro paciente que vai sair daqui com a qualidade de vida ou não modificada. A gente cuida da mesma maneira. (TE9).

Assim como a assistência ao paciente é fundamental, a importância da assistência à família também foi destacada pelos profissionais entrevistados. Esta relação também era percebida por eles como uma dificuldade no cuidado em UTI.

\section{Dificuldades no cuidado com a família do potencial doador de órgãos}

Para os participantes, o cuidado de enfermagem do paciente internado em UTI também era voltado para a família. Entretanto, percebe-se que os profissionais apresentavam dificuldades para lidar com essa família, devido ao impacto emocional provocado por esse possível diagnóstico.

Depende também o quanto a gente se envolve com a família, o quanto a família deixa transparecer [...] tem alguns [familiares] que desabam ali e precisam da gente e a gente até chora junto. (E1).

Eu não tenho problema de cuidar do paciente em morte encefálica. Eu tenbo problema em cuidar da família do paciente em morte encefálica. Então, eu acho que são coisas diferentes [...] eu percebo que tem uma barreira entre a família e a enfermagem [...] então, eu sinto muita falta disso... de dar o acolbimento e de acolher. (E6).

Alguns relatos apontam a necessidade de uma abordagem sensível da equipe de enfermagem com a família, para auxiliar a aceitação da morte encefálica e a compreensão da possibilidade e dos benefícios da doação de órgãos. 
Não é uma coisa esperada muitas vezes, não é um paciente que já estava doente [...] Então, é uma coisa traumática não só para... óbvio que, para o paciente, mas também para o familiar. E aquela expectativa de se realmente for diagnosticada a morte encefálica, como vai ser aceito e se entrará para o protocolo de doação. Se a pessoa é apta a doar, como os familiares vão aceitar. E as experiências que eu tenho são tristes. A maioria dos familiares não entende e não aceita doar os órgãos, quando é constatado. (TE4).

O que teve assim, que me deixou bem marcada, foi um paciente que teve internado aqui [...] a esposa dele queria doar e os familiares dele, não. Daí ela manejou, teve todo aquele cuidado, até convencer a família para doar os órgãos [...] mas é um trabalho fantástico, a maneira de tu abordar as pessoas que eu acho que é o mais dificil ali [...] tu tens que ter muita, muita firmeza no que tu vai falar, para tu não ferir as pessoas naquele momento. (TE8).

Por outro lado, a recusa do familiar para doação e, consequentemente, a retirada dos aparelhos também pode ser um momento emocionalmente impactante para a equipe de enfermagem, conforme se percebe no seguinte relato:

Teve uma vez que foi um guri bem jovem, e a família não quis doar. Dai a família entrou ali e o médico falou que ia desligar. Estava tudo certo, daí ele desligou o ventilador, a família ficou junto ali. Claro foi ruim ver a mãe chorando [...] só ficou aquela imagem. (TE11).

\section{Qualificação e preparo para o cuidado do paciente em morte encefálica}

A necessidade de qualificação para o trabalho de enfermagem, nesse contexto, é manifestada na fala de uma entrevistada. O aperfeiçoamento de competências precisa contemplar tanto os aspectos conceituais e clínicos relacionados à morte encefálica quanto a doação de órgãos, conforme os depoimentos que se seguem:

\begin{abstract}
Eu acho que a gente tem que se qualificar para a mor te encefálica, tanto quanto para a doação de órgãos, quanto para falar com a família, trabalbar em equipe [...] Acho tinha que ser falado sobre o luto; tinha que ser falado sobre o lidar com morte encefálica; tinha que preparar a gente para fazer um acolbimento melbor e qualificado com as familias, fortalecer, porque a gente sabe que existe [...] Então, para isso, a gente precisa estudar, se qualificar, motivação e liderança. Se a gente não tem essa equipe assim dentro de uma UTI, as pessoas se acomodam e não fazem a mudança necessária. (E6)
\end{abstract}

[...] eu vejo aqui colegas dizerem "ah, mas será que volta? Será que não volta?" (E5).

Eu sinto falta de conbecimento [...] porque ali existe uma vida que pode salvar muitas vidas. (E7).

Evidenciou-se que o cuidado de enfermagem ao paciente em suspeita ou com diagnóstico de morte encefálica exige dos trabalhadores habilidades cognitivas e emocionais, além de conhecimento científico e uma compreensão aprofundada da fisiopatologia dessa condição. Assim, percebe-se que o cuidado e o suporte, tanto para a família quanto para o paciente com suspeita ou diagnóstico de morte encefálica, são de extrema relevância, porém se constitui fonte de desgaste emocional para a equipe.

A expectativa com relação à doação de órgãos, muitas vezes, torna-se o enfoque que dá significado ao cuidado prestado ao paciente em morte encefálica. Os participantes do estudo relataram que tentavam canalizar os seus sentimentos para os aspectos positivos, como as vidas que poderão ser salvas, e não para a vida que ali se perdeu.

Eu acho que, assim, a morte encefálica tem os dois lados... pensando positivamente, a gente pensa no potencial doador. Então, ao mesmo tempo, acho que eu, pelo menos, foco na parte do potencial doador; que vai ajudar outras pessoas que precisam. (E2).

Mas eu acho que é bem assim. Um paciente morre, mas tu sabes que é outro que está nascendo, como se diz. (TE1).

Contribuir para uma outra vida. Pensar que se aquele ali não deu, para ele terminou ali, para uma outra vida pode ser uma continuidade, uma possibilidade. (E5).

Para esses profissionais, esse cuidado intenso ao potencial doador de órgãos pode se tornar gratificante frente à possibilidade de doação de órgãos. O trabalho é focado em viabilizar e garantir a qualidade dos órgãos que serão transplantados para aqueles que necessitam, para assegurar a continuidade da vida.

Se ele for doar os órgãos, e a gente tiver fazendo de tudo ali, cuidando, eu acho bem importante, me sinto muito bem. Ab, significa que ali ele vai estar ajudando outras vidas! (TE11)

Quando tem a possibilidade de doação de órgãos, tu exer ces um trabalbo pensando nisso. Que essa pessoa está ali com possibilidade de doar órgãos e salvar outras vidas. (E4).

No entanto, segundo os participantes, vários fatores interferem negativamente no cuidado ao paciente. O processo de doação de órgãos, por vezes demorado, acaba levando à negativa familiar, frustrando e desmotivando alguns profissionais, de acordo com as seguintes falas: 
Eu penso naquela coisa de doar os órgãos, que vai ajudar outras pessoas. Só que o processo ainda é muito atrasado, fica muita burocracia em volta. Claro que eu sei que isso faz parte. (TE11)

$A h$ às vezes atrasa. Se está chovendo eles [equipe de retirada de órgãos] não vão vir; se é tarde da noite, o avião não vai pousar. Já aconteceu de tudo. O problema é fora daqui que é complicado... (TE11).

A maioria das famílias desiste por causa do processo. (E2).

Os trabalhadores acabam criando a esperança da doação dos órgãos, pensando nas pessoas que aguardam na fila e que necessitam de um órgão novo para recuperar sua expectativa e qualidade de vida. No entanto, deparam-se com frustrações, como se apresenta nos depoimentos a seguir:

Aquele desejo que doem os órgãos, e a gente fica naquela expectativa, mas quase sempre frustrada que, no finalzinho, a família decide não doar. Daí tu vai cuidando dos órgãos, aquelas soluções para manter esses órgãos em bom estado, e chega no final e o familiar diz não. Então, é bem frustrante assim. (TE9).

É bem frustrante quando não acontece ou quando a família dá para trás, ou quando a família dá uma negativa de inicio. E esse paciente então vai a óbito. Geralmente são pacientes jovens. (E2).

Dessa forma, percebe-se que o cuidado ao paciente em diagnóstico de morte encefálica é complexo e pode despertar reações ambíguas nos trabalhadores, ora de frustração pela vida que se perdeu, ora de esperança pela possibilidade de salvar outras vidas por meio da doação de órgãos. Os resultados evidenciaram que os profissionais de enfermagem visualizavam esse processo para além da morte e do morrer, também como uma forma de renovação de outras vidas.

\section{Discussão}

A análise dos depoimentos possibilitou perceber que as questões que envolvem a morte, em particular as de ordem subjetiva, figuram entre as maiores dificuldades experimentadas pelos trabalhadores de enfermagem, pois a sua formação profissional tem sido voltada para o salvamento de vidas e evitar a morte com base nos conhecimentos técnicos e científicos. No entanto, quando se deparam com a terminalidade da vida, revela-se o sentimento de impotência e se torna evidente o despreparo desses profissionais para lidar com as perdas ${ }^{(2,16)}$.

Estudo $^{(6)}$ identificou que a dificuldade no cuidado ao paciente em morte encefálica ocorre na compreensão de que um paciente que está comprovadamente morto exige uma alta complexidade de cuidados dos profissionais de saúde. Os trabalhadores, quando questionados sobre a sua experiência no processo, relataram uma "mistura de sentimentos" e afirmaram que as emoções apareciam implícitas durante todo o processo de cuidado.

Quando existe a suspeita ou o diagnóstico de morte encefálica, é possível perceber uma mobilização de emoções, sentimentos e crenças de todos os envolvidos. Isso ocorre com amigos, familiares e até mesmo com os profissionais que atuam no processo de cuidado. Para estes últimos, que convivem cotidianamente com tal situação, é de suma importância que exista uma qualificação específica, visto que esse quadro clínico vem se tornando cada vez mais comum, devido ao aumento de morbimortalidade por causas externas (acidentes automobilísticos, vítimas de agressão, entre outros) ${ }^{(17)}$. A comoção e a dificuldade de aceitar esse processo foram percebidas nos depoimentos dos profissionais de enfermagem, no decorrer do presente estudo.

Pesquisa $^{(2)}$ aponta que os profissionais de saúde relatam sentimento de tristeza frente à perda de um paciente jovem, pelo fato de se tratar de uma morte inesperada, uma barreira que interrompe uma vida longa que havia pela frente. Dados epidemiológicos ${ }^{(18)}$ revelam que o perfil de paciente que vai a óbito, por causas externas, é majoritariamente de adulto jovem, o que vai ao encontro dos depoimentos dos profissionais entrevistados.

Uma questão conflitante para os enfermeiros entrevistados em outros três estudos ${ }^{(6,17,19)}$ estava relacionada à compreensão e ao aceite do diagnóstico da morte encefálica. Os resultados desta pesquisa apontam que os participantes também demonstraram essa sensação de ambiguidade ao lidar com a perspectiva de morte. Quando se trata de paciente com sinais vitais estáveis, também remete a um corpo vivo. Mesmo existindo um 
diagnóstico de morte, o cuidado precisa ser tão efetivo quanto o de um paciente vivo.

A importância da qualificação específica sobre morte encefálica, para a equipe de enfermagem de terapia intensiva, evidenciada nas falas dos entrevistados, é referida na literatura. O papel da enfermagem diante de paciente em morte encefálica na UTI é de grande importância, sendo fundamental que a equipe de enfermagem tenha conhecimento científico a respeito da fisiopatologia relacionada à morte encefálica, tendo em vista que exerce papel importante na monitorização do paciente, viabilizando o processo de doação de órgãos ${ }^{(8,20)}$. Também se destaca a necessidade de a equipe de enfermagem ter conhecimento das etapas que permeiam esse processo, a fim de que possam construir estratégias que auxiliem para que ele aconteça de forma mais eficiente $^{(20)}$.

A falta de conhecimento por parte dos profissionais, assim como evidenciado neste estudo, é descrita por alguns autores como um dos motivos para a não doação dos órgãos. A falta de conhecimento sobre a fisiopatologia da morte encefálica faz com que muitos profissionais não se sintam seguros para lidar com os familiares. Pesquisas ${ }^{(2,21)}$ apontam que existem poucas capacitações sobre o tema. Além disso, as instituições de ensino não ofertam esse tipo de aprofundamento durante a graduação. A educação profissional é a chave para a possibilidade de sucesso no processo de doações e transplantes, reafirmando, assim, a importância de treinamentos, palestras e cursos como fundamentais para potencializar a assistência ${ }^{(6)}$.

Além da qualificação profissional, é necessário que o enfermeiro esteja preparado para o adequado acolhimento à família dos pacientes que estão em processo de morte e morrer. Para alguns autores ${ }^{(6,21)}$, os enfermeiros devem ter ciência que prover o cuidado à família exige conhecimentos que vão além do saber técnico. Esse cuidado implica no compartilhamento de saberes, necessidades, possibilidades, angústias e invenções, sobretudo o cuidado no atendimento à família diante da dor e do sofrimento.
Diante da doença e na iminência da morte, os familiares dos pacientes nunca estão, de fato, preparados para a perda. Por isso, os profissionais de saúde acabam se tornando pilares para a família, e são considerados fundamentais nesse processo $^{(22)}$. Isso indica a necessidade do fortalecimento no acolhimento aos familiares de pacientes internados em UTI, especialmente para aqueles que têm a suspeita ou diagnóstico de morte encefálica.

A enfermagem é um campo profissional no qual seus trabalhadores atuam no cuidado direto ao paciente, nas 24 horas do dia. Por esse motivo, esses profissionais estão mais presentes e sentem-se ligados à família do potencial doador. Essa proximidade pode fazer com que essa equipe acabe mais envolvida com os aspectos emocionais dos familiares durante o processo de perda de um ente. Assim, necessitam de conhecimento, para que possam gerar segurança para a família que sofre e, ao mesmo tempo, possuem dúvidas por não compreenderem o que é realmente o processo de morte encefálica ${ }^{(2,21)}$.

De forma semelhante ao que foi verificado neste estudo, pesquisa ${ }^{(23)}$ realizada com profissionais de enfermagem demonstrou que eles acreditavam que a recusa por parte da família acabava dificultando a efetivação da doação de órgão. Os dados da Associação Brasileira de Transplantes de Órgãos ${ }^{(9)}$ mostram que 42\% das não doações ocorreram por recusa familiar, confirmando a importância desse aspecto na viabilidade da doação no Brasil. Sendo assim, configura-se a necessidade de serem prestadas informações a respeito da doação de órgãos para a sociedade.

O processo de transplante apresenta dois fatores essenciais: o primeiro, é a qualificação da equipe de saúde; e o segundo, a educação da comunidade. A educação permanente é fundamental, pois contribui para o aumento da qualidade do cuidado prestado aos potenciais doadores; os profissionais atuam de forma ágil no processo dos testes necessários para o diagnóstico de morte encefálica e podem melhorar 
a abordagem à família, influenciando diretamente na efetivação da doação ${ }^{(23)}$.

Conforme a Resolução n. 292/2004 do Conselho Federal de Enfermagem ${ }^{(24)}$, o papel do enfermeiro intensivista, que possui base legal para o cuidado, é o de ser responsável por planejar, coordenar, executar e avaliar as ações da equipe de enfermagem realizadas junto ao paciente em suspeita ou diagnóstico de morte encefálica, que podem ser potenciais doadores de órgãos e tecidos. Além do esclarecimento para a sociedade, também é necessário que os profissionais de saúde tenham maior conhecimento sobre o tema, visto que eles são um dos elos determinantes para o sucesso ou o fracasso dos programas de transplantes.

O processo de perda do paciente provoca sofrimento, porém a perspectiva da doação de órgãos proporciona conforto, pois, apesar de existir uma vida que está se perdendo, aqueles órgãos poderão salvar outras pessoas. O cuidado oferecido ao potencial doador, quando percebido como meio para salvar outras vidas, torna-se gratificante. Sendo assim, o processo de doação de órgãos, apesar de ser um trabalho desgastante e, por vezes, burocrático e demorado, apresenta-se como um aspecto positivo quando se trata do assunto de morte encefálica ${ }^{(2,6,17)}$.

O estudo apresenta limitações devido ao fato de possuir uma abordagem qualitativa da realidade local, não podendo, assim, ser feita a generalização dos resultados. Entretanto, o estudo contribui para o conhecimento acerca da percepção de enfermeiros no cuidado de pacientes em suspeita ou diagnóstico de morte encefálica em UTI.

\section{Conclusão}

Os resultados evidenciam que o cuidado da equipe de enfermagem ao paciente em morte encefálica está permeado por implicações relacionadas às dificuldades, tendo em vista que é desgastante psicologicamente e por se tratar de um paciente que requer monitorização contínua e atenção permanente. Além disso, é possível destacar a preocupação, o esforço e o cuidado dos trabalhadores de enfermagem para viabilizar os órgãos do paciente que está com suspeita ou diagnóstico de morte encefálica, pois ele pode tornar-se um futuro doador.

Os relatos dos trabalhadores evidenciam a vivência de contradições de uma relação de cuidado que envolve o enfrentamento da morte, mas, por outro lado, a possibilidade da promoção da vida por meio da doação dos órgãos. Ademais, a recusa familiar foi destacada como um fator importante na impossibilidade de doar os órgãos. Diante disso, salienta-se a necessidade de profissionais capacitados para realizar esse vínculo, visto que o cuidado com a família pode também ser utilizado para aprimorar o conhecimento da população acerca do tema.

Sendo assim, percebe-se que a equipe de enfermagem tem papel fundamental na prestação de cuidados de um paciente com suspeita ou diagnóstico de morte encefálica, na abordagem ao familiar, e também na ação de educação em saúde, o que pode viabilizar e aumentar o número de doações de órgãos no Brasil. Diante disso, recomenda-se a implementação de atividades educativas para esses profissionais acerca da temática e a possibilidade de inserção de apoio psicológico ao trabalhador frente à ambiguidade do processo de cuidado com este tipo de paciente. Também se sugere a implementação do tema na formação acadêmica, visando preparar os profissionais para a execução de atividades a ele relacionadas. Além disso, ressalta-se a importância da realização de outros estudos semelhantes em realidades diferentes, para aprofundamento do conhecimento na área. Por fim, informa-se que não houve conflitos de interesse para a realização deste trabalho.

\section{Colaborações:}

1 - concepção, projeto, análise e interpretação dos dados: Mariana Pellegrini Cesar e Quezia Boeira da Cunha;

2 - redação do artigo e revisão crítica relevante do conteúdo intelectual: Mariana Pellegrini Cesar, Quezia Boeira da Cunha, Camila Pinno, Nara 
Marilene Oliveira Girardon-Perlini, Cíntia Lovato Flores e Silviamar Camponogara;

3 - aprovação final da versão a ser publicada: Mariana Pellegrini Cesar e Silviamar Camponogara.

\section{Referências}

1. Longuiniere ACF, Lobo MP, Leite PL, Barros RCS, Souza AN, Vieira SNS. Conhecimento de enfermeiros intensivistas acerca do processo de diagnóstico de morte encefálica. Rev Rene. 2016;17(5):691-8. DOI: $10.15253 / 2175-6783.2016000500015$

2. Castro MFS, Rocha RLP, Fialho LP, Silva PAT, Oliveira RSP, Costa ML. Conhecimento e atitude dos enfermeiros frente ao processo de doação de órgãos. Rev Med Minas Gerais. 2018;28(5):e-S280504. DOI: $10.5935 / 2238-3182.20180016$

3. Brasil. Decreto n. 9.175, de 18 de outubro de 2017. Regulamenta a Lei n. 9.434, de 4 de fevereiro de 1997, para tratar da disposição de órgãos, tecidos, células e partes do corpo humano para fins de transplante e tratamento [Internet]. Brasília, DF; 2017 [cited 2019 Oct 10]. Available from: http://www.planalto.gov.br/ccivil_03/_ato20152018/2017/decreto/D9175.htm

4. Conselho Federal de Medicina. Resolução n. 2.173, de 23 de novembro de 2017. Define os critérios do diagnóstico de morte encefálica. Diário Oficial da União. Brasília, DF; 201715 dez; Seção 1, p. 50-275.

5. Cavalcante LP, Ramos IC, Araújo MAM, Alves MDS, Braga VAB. Cuidados de enfermagem ao paciente em morte encefálica e potencial doador de órgãos. Acta Paul Enferm. 2014;27(6):567-72. DOI: 10.1590/1982-0194201400092

6. Magalhães ALP, Erdmann AL, Sousa FGM, Lanzoni GMM, Silva EL, Mello ALSF. Significados do cuidado de enfermagem ao paciente em morte encefálica potencial doador. Rev Gaúcha Enferm. 2018;39:e2017-0274. DOI: 10.1590/1983-1447.2018.2017-0274

7. Victorino JP, Ventura CAA. Doação de órgãos: tema bioético à luz da legislação. Rev bioét. 2017;25(1): 138-47. DOI: 10.1590/1983-80422017251175

8. Costa CR, Costa LP, Aguiar N. A enfermagem e o paciente em morte encefálica na UTI. Rev bioét. 2016;24(2):368-73. DOI: $10.1590 / 1983-80422016242137$
9. Associação Brasileira de Transplante de Órgãos. Dimensionamento dos transplantes no Brasil e em cada estado 2018. Registro Brasileiro de Transplantes [Internet]. São Paulo; 2018 [cited 2019 Oct 10];XXIV(4). Available from: http://www.abto. org.br/abtov03/Upload/file/RBT/2018/Lv_RBT2018.pdf

10. Costa CR, Costa LP, Aguiar N. A enfermagem e o paciente em morte encefálica na UTI. Rev bioét. 2016;24(2):368-73. DOI: 10.1590/1983-80422016242137

11. Flodén A, Berg M, Forsberg A. ICU nurses' perceptions of responsibilities and organisation in relation to organ donation: a phenomenographic study. Intensive Crit Care Nurs. 2011;27(6):305-16.

12. Minayo MCS. Amostragem e saturação em pesquisa qualitativa: consensos e controvérsias. Rev Pesq Qualitativa. 2017;5(7):1-12. DOI: 10.33361/ RPQ.v.6.n.12

13. Bardin L. Análise de conteúdo. 7a ed. São Paulo: Edições 70; 2011.

14. Conselho Nacional da Saúde. Resolução n. 466, de 12 de dezembro de 2012. Aprova diretrizes e normas regulamentadoras de pesquisas envolvendo seres humanos [Internet]. Brasília, DF; 2012 [cited 2019 Oct 10]. Available from: https:// conselho.saude.gov.br/resolucoes/2012/Reso466. pdf

15. Tong A, Sainsbury P, Craig J. Consolidated criteria for reporting qualitative research (COREQ): a 32item checklist for interviews and focus groups. Int J Qual Health Care. 2007;19(6):349-57.

16. Menin GE, Pettenon MK. Terminalidade da vida infantil: percepções e sentimentos de enfermeiros. Rev bioét. 2015;23(3):608-14. DOI: 10.1590/1983-80422015233097

17. Almeida EC, Bueno SMV, Baldissera VAD. Atuação de profissionais de saúde em doação de órgãos na perspectiva do familiar: uma análise problematizadora. Arq Cienc Saúde UNIPAR. 2015;19(2):139-45. DOI: 10.25110 /arqsaude. v19i2.2015.5434

18. Brasil. Ministério da Saúde. Taxa de mortalidade específica por causas externas [Internet]. Brasília, DF; 2012 [cited 2019 Apr 22]. Available from: http:// tabnet.datasus.gov.br/cgi/tabcgi.exe?idb2012/c09. def

19. Keshtkaram Z, Sharif F, Navab E, Gholamzadeh $S$. Lived experiences of Iranian nurses caring for brain death organ donor patients: caring as 
"Halo of ambiguity and doubt". Glob J Health Sci. 2016;8(7):281-92. DOI: 10.5539/gjhs.v8n7p281

20. Souza BSJ, Lira GG, Mola R. Notificação da morte encefálica em ambiente hospitalar. Rev Rene. 2015;16(2):194-200. DOI: 10.15253/2175-67 83.2015000200008

21. Fernandes MEN, Bittencourt ZZLC, Boin IFSF. Vivenciando a doação de órgãos: sentimentos de familiares pós consentimento. Rev Latino-Am Enferm. 2015;23(5):895-901. DOI: http://dx.doi. org/10.1590/0104-1169.0486.2629

22. Santana JCB, Dutra BS, Carlos JMM, Barros JKA. Ortotanásia nas unidades de terapia intensiva: percepção dos enfermeiros. Rev bioét. 2017;25(1):158-67. DOI: 10.1590/1983-8042201725 1177
23. Costa IF, Mourão Netto JJ, Brito MCC, Goyanna NF, Santos TC, Santos SS. Fragilidades na atenção ao potencial doador de órgãos: percepção de enfermeiros. Rev bioét. 2017;25(1):130-7. DOI: 10.1590/1983-80422017251174

24. Conselho Federal de Enfermagem. Resolução n. 292, de 7 de junho de 2004. Normatiza a atuação do Enfermeiro na Captação e Transplante de Órgãos e tecidos [Internet]. Brasília, DF; 2004 [cited 2019 Oct 10]. Available from: http://www.cofen.gov.br/ resoluo-cofen-2922004_4328.html

Recebido: 29 de agosto de 2019 Aprovado: 2 de novembro de 2019

Publicado: 19 de dezembro de 2019

A Revista Baiana de Enfermagem utiliza a Licença Creative Commons - Atribuição-NãoComercial 4.0 Internacional. https://creativecommons.org/licenses/by-nc/4.0/

Este artigo é de acesso aberto distribuído sob os termos da Licença Creative Commons (CC BY-NC). Esta licença permite que outros remixem, adaptem e criem a partir do seu trabalho para fins não comerciais. Embora os novos trabalhos tenham de lhe atribuir o devido crédito e não possam ser usados para fins comerciais, os usuários não têm de licenciar esses trabalhos derivados sob os mesmos termos. 